\title{
A Novel Approach to in situ Proteome Analysis Using Chemical Ink jet Printing Technology and MALDI-QIT-TOF Tandem Mass Spectrometer
}

\author{
Shuichi Shimma, ${ }^{1,2}$ Masaru Furuta, ${ }^{3}$ Katsuhiko Ichimura, ${ }^{3}$ \\ Yoshikazu Yoshida, ${ }^{4}$ and Mitsutoshi Setou ${ }^{1,2 *}$ \\ ${ }^{1}$ Okazaki Institute for Integrative Bioscience, National Institutes of Natural Sciences, \\ Okazaki, AICHI, JAPAN \\ ${ }^{2}$ School of Life Science, The Graduate University for Advanced Studies, Okazaki, \\ AICHI, JAPAN \\ ${ }^{3}$ Life Science Business Unit, Analytical \& Measuring Instruments Division, Shimadzu \\ Corporation, Kyoto, JAPAN \\ ${ }^{4}$ Technology Research Laboratory, Shimadzu Corporation, Seika, Soraku-gun,
} KYOTO, JAPAN

\begin{abstract}
Proteomics data obtained by mass spectrometry are now being combined with spatial information. This report outlines a digestion procedure for tissue sections on polyvinylidenfluoride membrane and results of a direct tandem mass spectrometry of mouse brain sections in situ. We succeeded in sequencing the digested peptides such as myelin basic protein, histon $\mathrm{H} 2 \mathrm{~A}$, and tubulin $\beta 4$ directly from tissue sections and transferred membrane. Protein blotting method can provide protein denaturation during the transfer process with electrode heat and with detergent contained in a transfer buffer. In addition to denaturing proteins, chemical inkjet printing technology and a highly sensitive matrix-assisted laser desorption/ionization quadrupole-ion-trap time-of-flight tandem mass spectrometer enabled us to print solutions of enzyme in micro-scale areas on the membranes and obtain high quality data in mass spectrometry/mass spectrometry. This analytical methodology will provide an innovative approach to in situ proteome analysis.
\end{abstract}

(Received January 20, 2006; Accepted April 17, 2006)

\section{Introduction}

The direct molecular analysis of biological tissue using mass spectrometry (MS) is a subject of much interest for the next generation of mass spectrometry. ${ }^{1)}$ Mass spectra from tissue sections have already been shown to provide useful biological information.

Recent studies based on this new technique have achieved successful molecular imaging ${ }^{2)-6)}$ for biological applications, ${ }^{7-10)}$ pathological applications, ${ }^{1)^{-18)}}$ and drug discovery. ${ }^{19), 20)}$ For example, in a study of Alzheimer's disease, Stoeckli and co-workers have successfully mapped amyloid peptides. ${ }^{11), 16)}$ Such studies were developed using matrix-assisted laser desorption/ ionization (MALDI) time-of-flight (TOF) mass spectrometers. In addition to the MALDI approach, secondary ion mass spectrometry has also been used in the analysis of low molecular weight ions, e.g., phospholipids and cholesterol. ${ }^{21)-23)}$

However, the single-stage MS data of tissue sections do not provide direct information regarding the identi-

* Correspondence to: Mitsutoshi Setou, Okazaki Institute for Integrative Bioscience, National Institutes of Natural Sciences, Higashiyama 5-1, Myodaiji-cho, Okazaki, AICHI 444-8787, JAPAN, e-mail: setou@nips.ac.jp fication of biomolecules. Using a typical proteomics approach, the tandem MS data of peptides digested by enzymes such as trypsin enables the determination of amino acid sequences. Therefore, it is necessary to establish methods for digestion of tissue sections while at the same time retaining information regarding the relative positions of biomolecules. A technique of highly efficient mass spectrometry/mass spectrometry (MS/MS) is also required, because the ionization efficiency of the tissue surface is lower than that of purified analytes. To fulfill the above requirements, we used a chemical inkjet printer (CHIP-1000) ${ }^{24)-26)}$ and a MALDI quadrupole-ion-trap time-of-flight (MALDIQIT-TOF) tandem mass spectrometer. ${ }^{27)}$

In this paper, we establish a methodology for the MS/MS of tissue sections. We examined the methodology in the hippocampal region. We also explain the reason why we used the combination of CHIP-1000 and MALDI-QIT-TOF mass spectrometer for analysis of tissue sections.

\section{Experimental}

\section{Reagent}

Ammonium hydrogencarbonate, 2-propanol, acetonitrile, and trifluoroacetic acid (TFA) were purchased from Kanto Chemical Co., Inc. (Tokyo, Japan). Direct 
Blue 71 (DB 71) for membrane and tissue staining was purchased from Aldrich (St. Louis, MO, USA). Glycine and polyvinylpyrrolidone (PVP) were purchased from Sigma (St. Louis, MO, USA). SDS and 1,4-dithio-DLthreitol (DTT) were purchased from Research Organics (Cleveland, OH, USA). The Immobilon- $\mathrm{P}^{\mathrm{SQ}}$ transfer and tissue support membranes used in the present study were obtained from Millipore (Bedford, MA, USA). Sequencing-grade modified trypsin was purchased from Promega (Madison, WI, USA). Calibration-standard peptide mixture (1000-4000 Da) and 2,5-dihydroxybenzoic acid (DHB) were purchased from Bruker Daltonics (Leipzig, Germany).

\section{Mouse brains}

This study used 8-week-old male C57BL/6Cr mice. The mice were purchased from Japan SLC, Inc. (Shizuoka, Japan). The mice were sacrificed and dissected under diethyl ether anesthesia. The extirpated brains were immediately frozen in powdered dry ice and stored at $-80^{\circ} \mathrm{C}$ until needed.

\section{Apparatus}

Digestion of tissue sections and matrix dispensing were performed using a Chemical Inkjet Printer (CHIP1000). The instrument was developed by the Shimadzu Corporation (Kyoto, Japan) in collaboration with Proteome Systems, Ltd. (Sydney, Australia). ${ }^{26)}$ The CHIP1000 system could perform dispensing solutions in micro-scale areas of the tissue sections. Mass spectrometry was performed using a MALDI-QIT-TOF type instrument, the AXIMA-QIT (Shimadzu Corporation, Kyoto, Japan and Kratos Analytical, Manchester, UK), which was equipped with a 337-nm nitrogen laser. The repetition rate was optimized at $5 \mathrm{~Hz}$ due to QIT. The Data acquisition was performed in the positive-ion mode using an external calibration method with a peptide mixture of Angiotensin II $\left([\mathrm{M}+\mathrm{H}]^{+}: 1046.54\right)$ and ACTH clip 18-32 $\left([\mathrm{M}+\mathrm{H}]^{+}:\right.$2465.20).

\section{Preparation of tissue sections}

Frozen sections were sliced at $-15^{\circ} \mathrm{C}$ on a cryostat (Leica CM 3050) at a thickness of $30 \mu \mathrm{m}$. In order to fix the cutting block, an optimum cutting temperature (OCT) polymer was used. When the sections were sliced, the cutting block was not embedded in OCT, because any residual polymer on the tissue sections might have degraded the mass spectra. ${ }^{10)}$ The membranes were soaked in methanol for $30 \mathrm{~s}$ and immersed in water for $2 \mathrm{~min}$. The wetting membranes were maintained under vacuum conditions until they were used. After dehydration, frozen sections were thawmounted on the membranes.

\section{Protein blotting and staining of tissue sections}

The tissue sections on the dried membrane were transferred to another membrane. The transfer protocol followed the semi-dry electroblotting method. The capture membrane and filter pads were soaked in transfer buffer $(12.5 \mathrm{mM}$ Tris, $96 \mathrm{mM}$ glycine with 0.01\% SDS). ${ }^{11)}$ To perform disulfide bond cleavage, 50 $\mathrm{mM}$ of DTT was added to the transfer buffer in our protocol. The transfer was carried out under constant current conditions $\left(2.0 \mathrm{~mA} / \mathrm{cm}^{2}\right.$, depending on membrane size) for $30 \mathrm{~min}$. After blotting, both sections on membranes and transferred membranes were dyed with DB 71 and washed with 40\% ethanol and 10\% acetic acid for 5 min 3 times. The staining protocol followed a previously described method. ${ }^{28)}$ Stained sections and membranes were sufficiently dried in vacuum desiccators.

\section{On-membrane digestion}

A digestion protocol for blotted PVDF membrane after gel electrophoresis ${ }^{25)}$ was applied for the treatment of membranes and sections using the CHIP-1000 system. Conductive tape was used for the adhesion of the dried membranes to the MALDI target plate. The amount of $7 \mathrm{~nL}$ PVP solution in 60\% methanol $(0.25 \%$ $(\mathrm{w} / \mathrm{v}))$ was printed onto each transferred membrane for pre-wetting. The solution was printed as 7 iterations onto the surface at $1 \mathrm{~nL}$ per iteration. Sections on membranes and pre-wetted membranes were digested using $30 \mathrm{~nL}$ trypsin at $200 \mu \mathrm{g} / \mathrm{mL}$ in $25 \mathrm{mM} \mathrm{NH}_{4} \mathrm{HCO}_{3}$ containing $10 \%(\mathrm{v} / \mathrm{v}) 2$-propanol. After printing, the target plate was incubated for $12 \mathrm{~h}$ at $37^{\circ} \mathrm{C}$ under humid conditions, and then $100 \mathrm{~nL}$ of DHB $(8 \mathrm{mg} / \mathrm{mL}$ in $25 \%$ acetonitrile containing $0.1 \% \mathrm{TFA}$ ) was printed at each position. Printing procedure of trypsin and matrix solution was the same as the PVP solution.

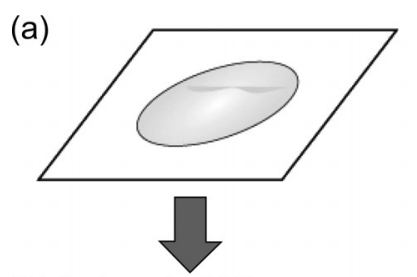

(b) Automated Dispenser

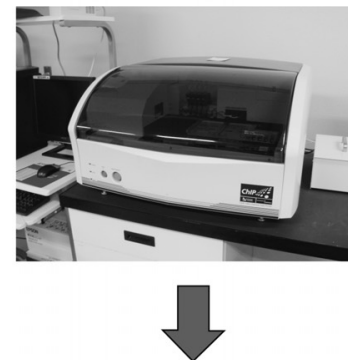

(c) MALDI-QIT-TOFMS

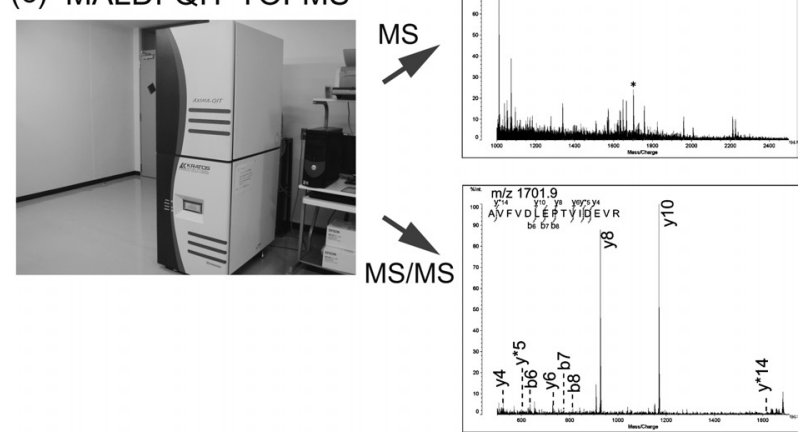

Fig. 1. Schemes of our strategy. (a) Frozen sections are thaw-mounted onto a PVDF membrane. Each section is transferred onto another PVDF membrane. Both the transferred membranes and the sections are stained with DB71. (b) Microscale printing is performed using a chemical inkjet printer. Stained membranes and sections are digested in a micro-scale area. (c) Positions of printed areas are analyzed by MALDI-QIT-TOF mass spectrometer. Because of QIT, MS/MS data are provided with high efficiency. 


\section{MS and MS/MS}

The data provided in this article were acquired using the AXIMA-QIT mass spectrometer. Acquisition was performed in the mid-mass range mode (750 Da to 3000 $\mathrm{Da})$ under $60 \mathrm{~V}$ of stage bias voltage. All mass spectra shown in the result section accumulated a total of 1000 profiles (2 laser shots/profile). Identification based on the MS/MS data was carried out using MS/MS ion search in Mascot (Matrix Science, Inc.).

\section{Results and Discussion}

\section{Scheme of our strategy}

The scheme of in situ proteome analysis is shown in Fig. 1. Sliced tissue section was mounted on the PVDF membrane (Fig. 1a). This section was transferred onto another PVDF membrane, and they were stained with DB 71. The digestion enzyme and matrix were dispensed onto the sample surface by CHIP-1000 (Fig. 1b). MS and MS/MS data were obtained with the AXIMAQIT (Fig. 1c). The sample preparation took about $13 \mathrm{~h}$.

\section{Staining and digestion}

To visualize the structure of brain sections, we stained both electroblotted membranes and sections with DB 71.28) Staining intensity was able to be controlled by the duration of exposure to the stain. Good contrast was obtained by $2.5 \mathrm{~min}$ for the recognition of the structures in tissue sections and transferred membranes (Figs. 2a and 2b).

Transferred membranes maintained the precise structure of the corresponding tissue sections. Moreover, the transfer process facilitated the denaturation as a result of heat from blotting electrodes and interactions between proteins and detergents in solution transfer buffer (SDS). Reduction by DTT was also achieved. Thus, the efficiency of tryptic digestion in both sections and transferred membranes was improved.

We can see the spatial compartmentation of brains, such as white matter, grey matter, hippocampus and cortex both in the transferred membranes and residue brain sections. Thus, the positional information of proteins was retained in a macroscopic level, even if sections and transferred membranes were washed many times. However, we have to test more carefully regarding this "migration" possibility in the case of higher resolution analysis in the future with mass microscopy.

According to the present method, microdispensing the reagents onto the sections and membranes was performed using CHIP-1000. The droplet volume required to achieve stable printing was $87 \mathrm{pL}$, and therefore we iterated the printing process to meet the desired quantity. As a result of this iteration process, the diameter of the printed area was $500 \mu \mathrm{m}$ (Figs. 2c and 2d).

Numerous areas on the tissue sections can be printed by optimizing the printing conditions in the same way according to the approach described in the experimental section. These homogeneous spots are useful for performing molecular imaging studies, because the matrix coated by spray is associated with the ionization efficiency due to the lack of uniformity of a matrix concentration on the tissue surface.

\section{Single-stage mass spectrometry}

The single-stage MS data in Figs. $3 \mathrm{a}$ and $3 \mathrm{~b}$ represent the average of 1000 profiles in each spot. Each spectra represents the position difference of laser irradiation. The region in the low $m / z$ area became noisy. The parameters of the procedure, including the laser intensity and the voltage applied to the sample plate, did not
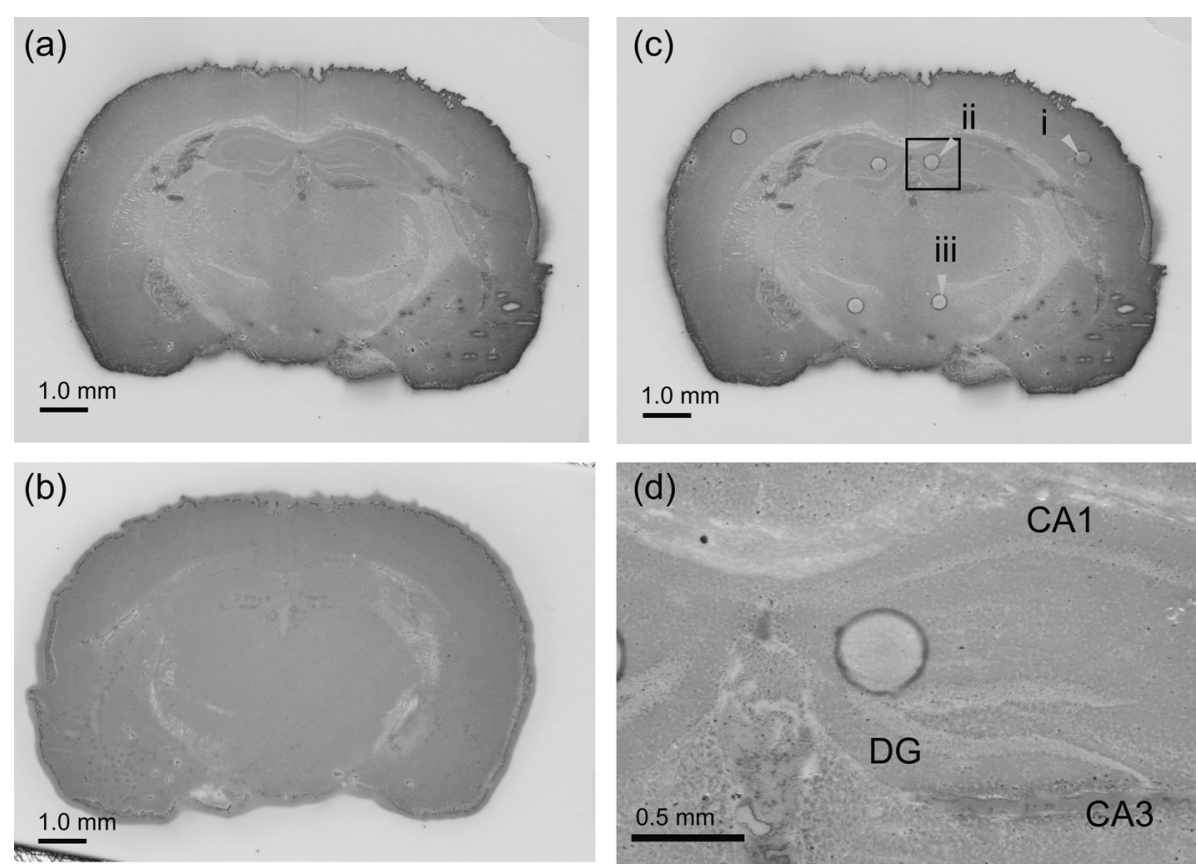

Fig. 2. Photomicrographs of $30 \mu \mathrm{m}$ coronal sections of mouse brain (bregma $-6.8 \mathrm{~mm}$ ). (a) A brain section stained by DB 71 after electroblotting shows the structure of the brain. (b) A blotted PVDF membrane also reveals the structure of the brain. (c) White dots indicate the printed areas of the reagents. Only 2,5-DHB was printed in the dashed circle to perform the control experiment. This image represents a stained section. (d) Enlarged view of the hippocampal region enclosed in (c). The characteristic structures of the hippocampus were clearly stained. 

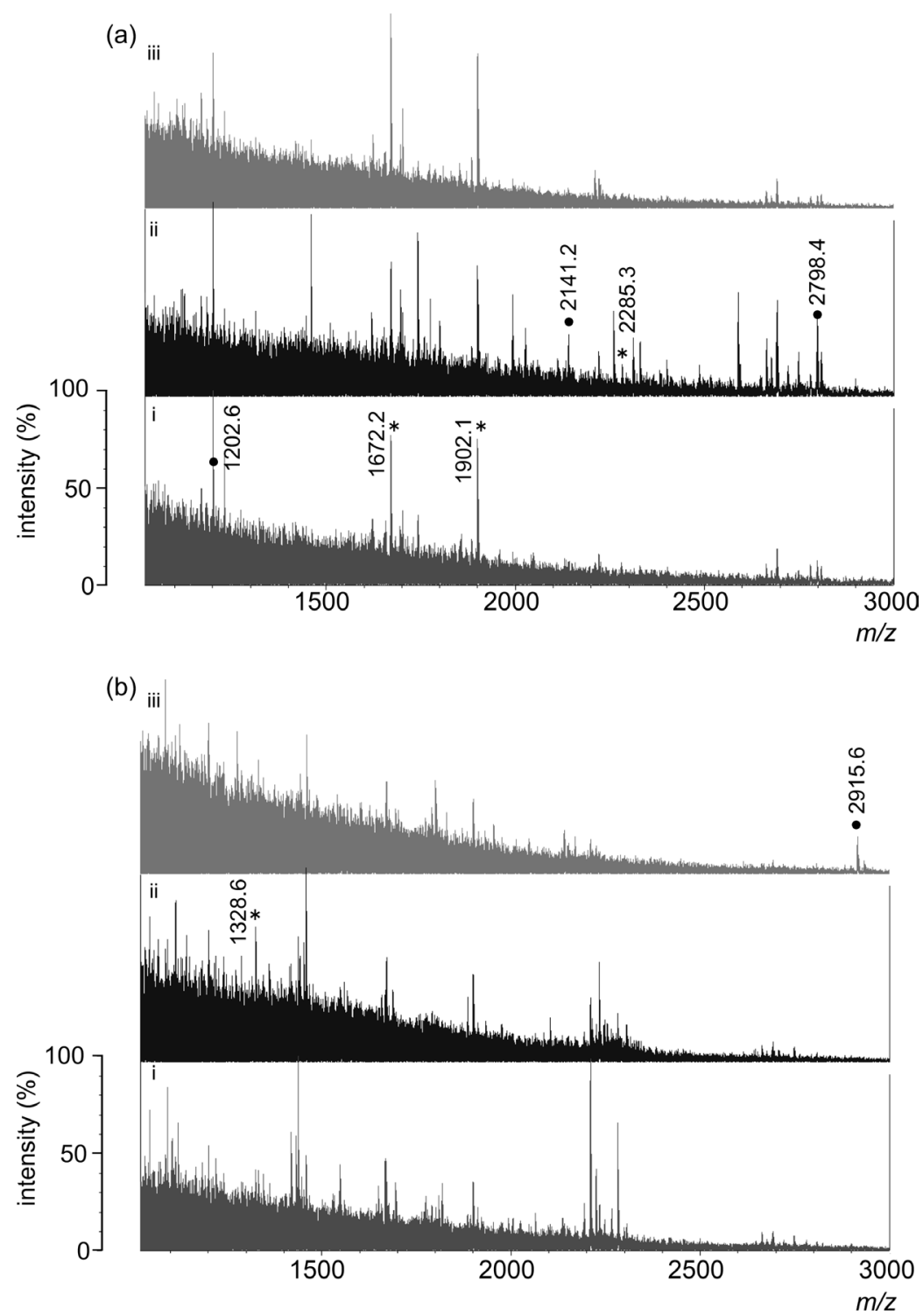

Fig. 3. The mass spectra obtained from mouse brain sections and transferred membranes. Single-stage MS data of stained tissue section (a) and transferred membrane (b). Each Roman numeral in the spectra indicates the position of laser irradiation represented in Fig. 2. We obtained MS/MS data from marked signals. Circle and asterisk indicate that identifications were available and unavailable, respectively.

affect the quality of the obtained spectrum. Such high noise level was due to low ionization efficiency on the tissue surface and PVDF surface. On the other hand, the detergent used for electroblotting did not interfere with any of the spectra, because repeated washing of the section and membrane with $40 \%$ ethanol and 10\% acetic acid removed the residual chemicals.

The control spectrum was derived from a dashed circle in Fig. 2c. We spotted only the matrix in this circle, so that this area reflected a lack of trypsin. The spectrum had no significant peaks above the noise level (Fig. 4). Compared to the control spectra, the tryptic digestion spectra were obtained from both tissue sections and transferred membranes (Fig. 3). A large number of peaks could be observed in the digested areas. High-intensity peaks, e.g., $m / z 1202,1672$, and 1902 (Fig. 3a), and 2915 (Fig. 3b), may indicate ubiquitous and abundant proteins. Therefore, it was concluded that the resulting signals were derived from digested products of proteins. After electroblotting, staining, and repeated washing processes, all lipid molecules and low molecular weight peptides might be removed.

\section{MS/MS data and identification}

We successfully obtained MS/MS data and identified molecules with ion signals at $m / z 2141.2$ (Fig. 5a), 2915.6 (Fig. 5b), 2798.4 (Fig. 5c), and 1202.6 (Fig. 5d). A Mascot-MS/MS ions search identified the following peptides: myelin basic protein (MBP) (Fig. 5a), histone H2A (Fig. 5b), and tubulin $\beta 4$ (Figs. $5 \mathrm{c}$ and $5 \mathrm{~d}$ ). The corresponding sequences and mass accuracies are also shown in each spectrum. The explanations of identified protein are as follows. MBP is composed of the myelin sheath which plays a role of insulator for axons. $^{29)}$ Histones are the chief proteins of chromatin. They act as spools around which DNA winds and they play a role in gene regulation. ${ }^{30)}$ The $\alpha \beta$-tubulin heterodimer of $110 \mathrm{kDa}$ is a unit of microtubule which is one of the cytoskeltons. ${ }^{31)}$

Each spectrum contained peaks which could not be 


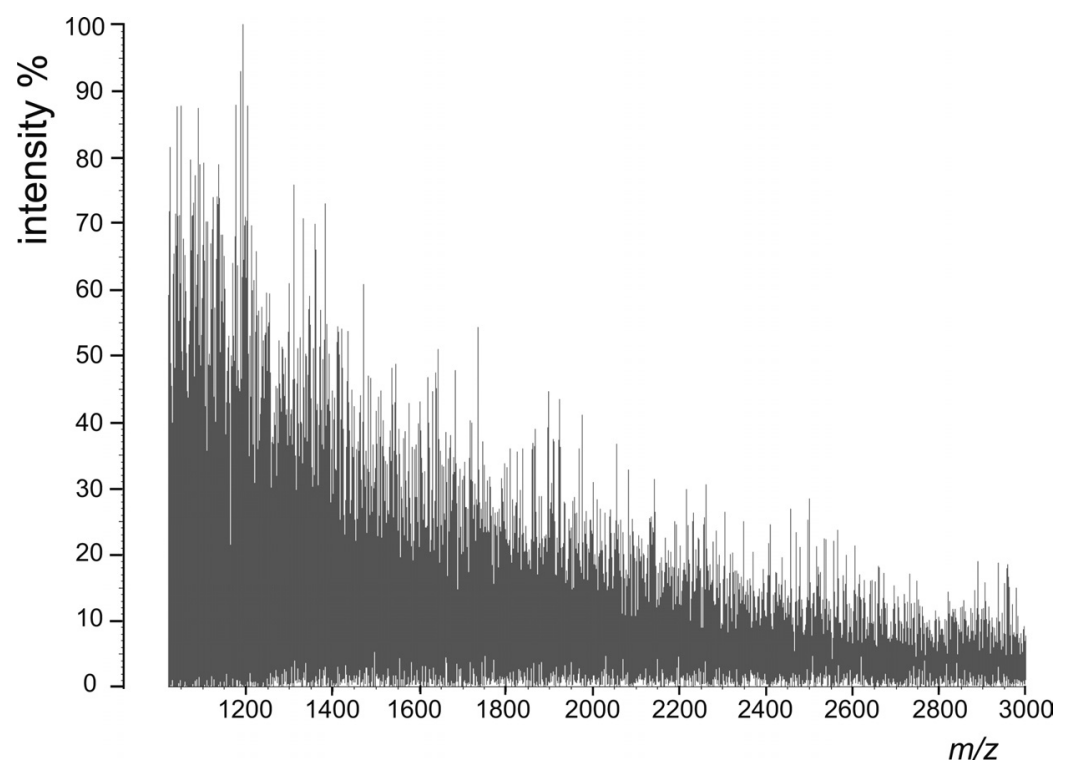

Fig. 4. The MS data from a control experiment accumulated 1000 profiles.

(a)

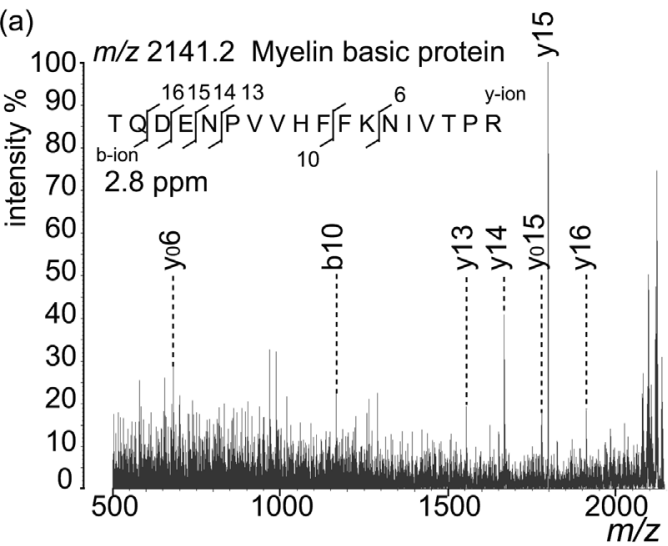

(c)

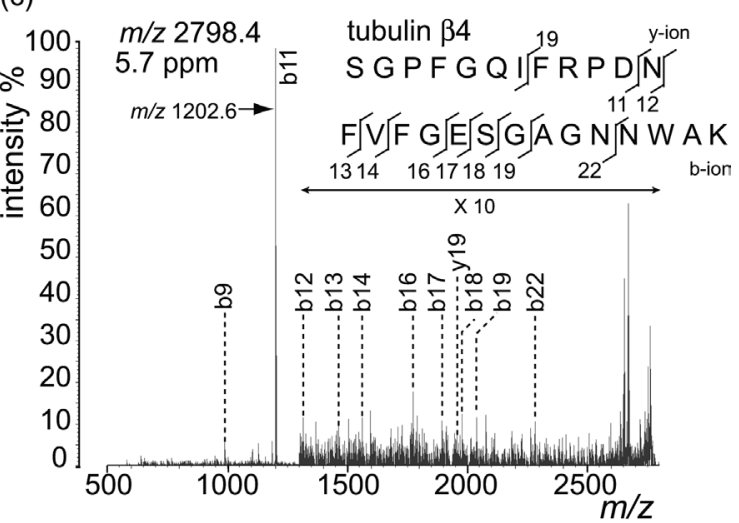

(d)

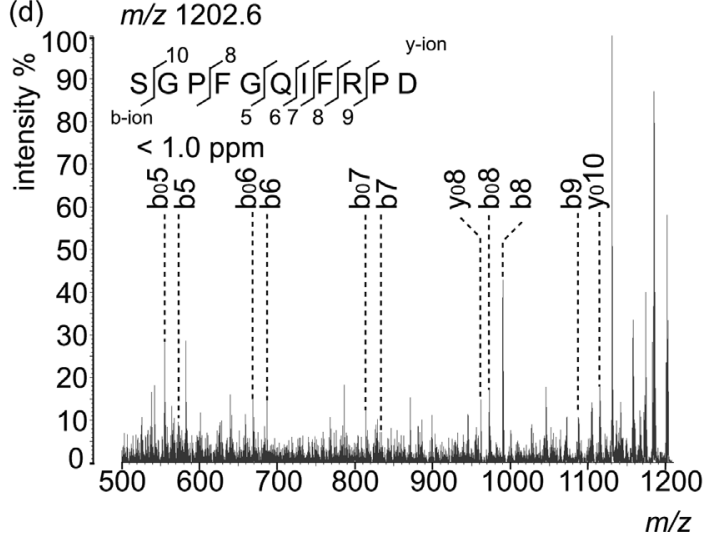

Fig. 5. MS/MS data obtained from the peaks (circle symbol) for Fig. 3. (a), (b) Product ion mass spectra of the $m / z 2141.2$ ion and 2915.6 ion were used to identify the precursor ions as myelin basic protein and histon H2A, respectively. (c) Product ion mass spectrum of the $\mathrm{m} / \mathrm{z} 2799.4$ revealed a high-intensity peak at $\mathrm{m} / \mathrm{z} 1202.6$, which was also noted in Fig. 3. The peak at $m / z 1202.6$ was derived from a fragment by in-source and/or post-source decay. (d) Product ion spectrum of the $\mathrm{m} / z 1202.6$ contained the same information as the $\mathrm{MS}^{3}$ data at $\mathrm{m} / z$ 2799.4. Considering (c) and (d), the ion at $m / z 2799.4$ were tubulin $\beta 4$.

assigned as fragments of the precursor ion. The spectra of the tissue sections had substantially different features from those of purified peptides. Thus, it appears that tissue sections provide a crude sample for mass spectrometry. The unassigned peaks shown in Fig. 5 appeared to be derived from the peripheral peaks of signals of interest. We could select 4 types of mass window which was represented as Low, Normal, High, and Extra High. The separation ability of QIT in the current setup is shown in Fig. 6 . In this figure, we presented the example of $m / z 1202.6$ because we would like to achieve a robustness test in the noisy region. 


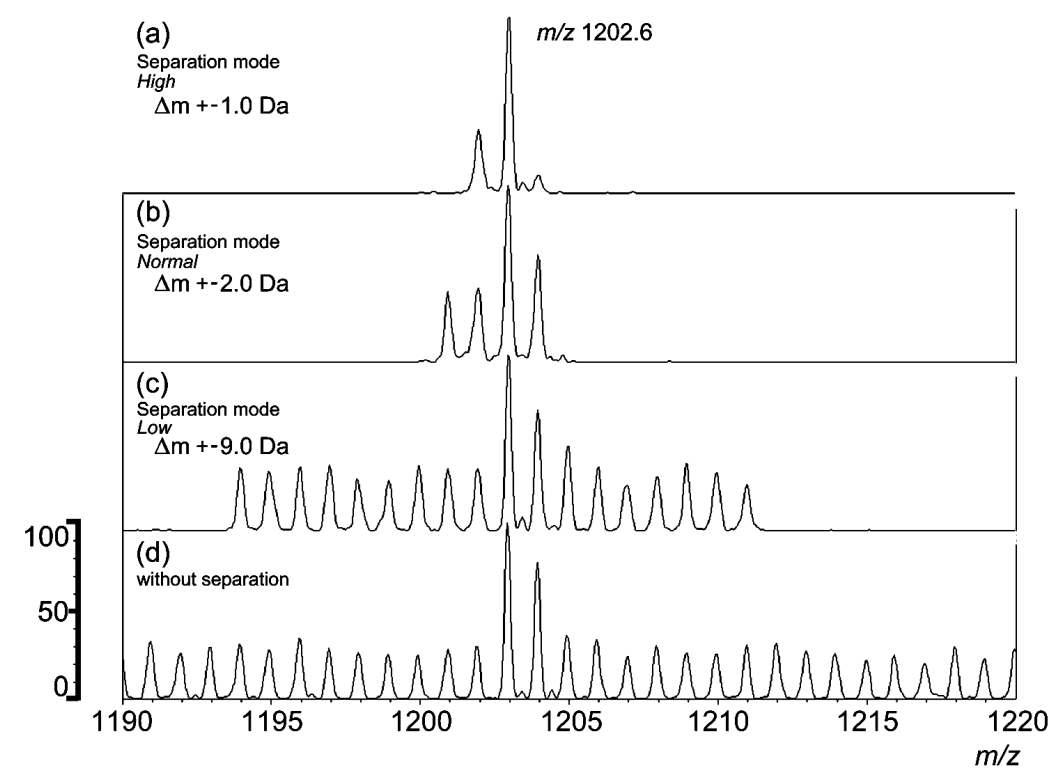

Fig. 6. Separation ability of QIT: The separation modes were High (a), Normal (b), Low (c), and Control (d).
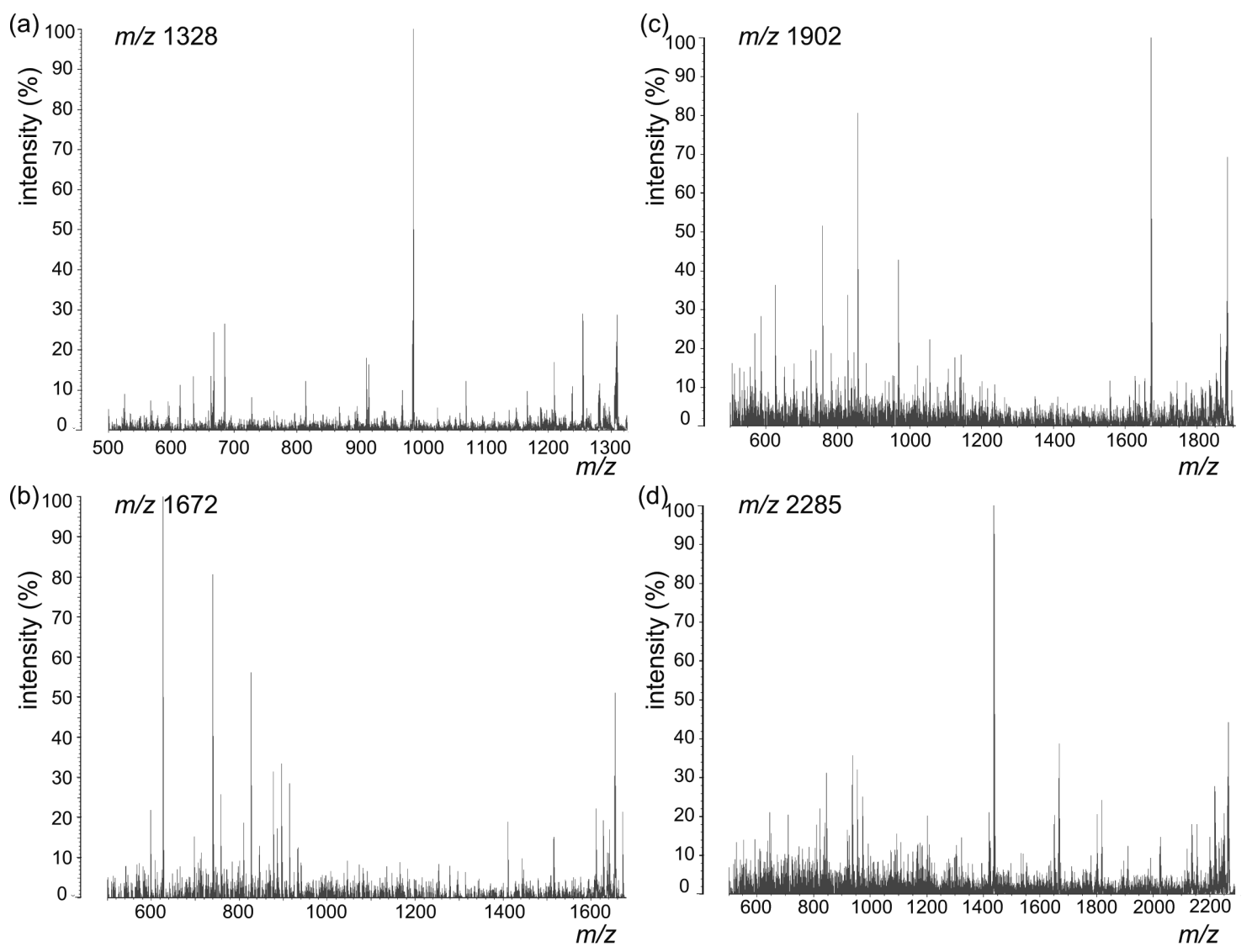

Fig. 7. Product ion mass spectra of the $m / z$ : (a) 1328 , (b) 1672 , (c) 1902 , and (d) 2285 . These spectra showed numerous high-intensity peaks. In the present study, we were unable to determine the amino acid sequences of the corresponding peptides.

The data in Extra High mode was omitted because the sensitivity in the Extra High mode was too low to measure on the tissue section. The best mass separation ability was 1.0 Da, however, MS/MS data in Fig. 5 was obtained in the Normal mode to keep sensitivity. Therefore the mass window was typically about 2.0 Da in this study.

It should be noted that the product ion mass spectrum at $m / z 1202.6$ was equivalent to the $\mathrm{MS}^{3}$ spec- trum at $m / z 2798.4$. The ion signal observed at $m / z$ 1202.6 also appeared as a fragment peak at $m / z 2798.4$, as shown in Fig. 5c. This result was considered to be an interesting feature obtainable by AXIMA-QIT. The ions generated by MALDI are transferred slowly to QIT with low voltage which is typically $60 \mathrm{~V}$ in this study. In this ion trap, energetic cooling is performed by helium for $150 \mathrm{~ms}$. During these processes, ions whose lifetime is shorter than the period of the process 
are dissociated naturally, and the fragment ions are observed in the single-stage MS data. This feature reflects both an advantage and a shortcoming of the present method. The advantage is that it may be easy to obtain $\mathrm{MS}^{3}$ data. We call such spectra $\mathrm{MS}^{3}$-like data. The example of this phenomenon is shown in Fig. $5 \mathrm{~d}$. The spectrum of $m / z 1202.6$ is $\mathrm{MS}^{3}$-like data of $\mathrm{m} / z$ 2798.4. The shortcoming is that undesired peaks can render the resulting spectra rather complex. The identification of tubulin $\beta 4$ represents an example of the benefits of this system. We suggest that the appearance of fragment peaks in the single-stage MS data is a powerful advantage in the analysis of post-translational modifications of peptides with known sequences, as $\mathrm{MS}^{3}$-like data can help to determine the sites of modification.

We obtained the MS/MS data of the additional peaks (asterisk) shown in Fig. 3. These additional spectra are shown in Fig. 7. Although these latter spectra revealed a number of high-intensity peaks and only a small amount of noise, the sequences of the corresponding molecules could not be determined by Mascot-MS/MS ion search. It appears that these spectra contain the $\mathrm{MS}^{3}$ information like Fig. 5d. Other explanations for the presence of these unknown peaks might have been post-translational modifications and/or peptides not recorded in the database yet.

\section{Conclusion}

We have successfully carried out the direct identification of digested products by observing the MS/MS data of mouse brain sections. Future development of this approach is the direct molecular imaging of microscale areas, as the microscopic identification of molecules via an approach designated as "MS microscopy." When we observe specimens, we have usually used conventional methodologies for microscopy, for example, fluorescent antibody method, ${ }^{32)}$ electron microscopy, ${ }^{33), 34)}$ and fluorescent protein labeling. ${ }^{35)}$ These techniques are good to observe structures, but not good for identification of molecules which form biological structures. Now, we are developing an instrument to realize $\mathrm{MS}^{n}$ for identification under observation with a microscope. $^{36), 37)}$

\section{Acknowledgements}

We would like to thank Y. Fukazawa and R. Shigemoto for their technical support of the cryostat operations. We also thank all members of the SENTAN project for their useful comments and discussions. This study was supported by a SENTAN grant-in-aid for Mitsutoshi Setou.

\section{References}

1) S. Shimma, Y. Yoshida, and M. Setou, J. Mass Spectrom. Soc. Jpn., 53, 230 (2005).

2) P. Chaurand, S. A. Schwartz, M. L. Reyzer, and R. M. Caprioli, Toxicol. Pathol., 33, 92 (2005).

3) R. L. Caldwell and R. M. Caprioli, Mol. Cell. Proteomics, 4, 394 (2005).

4) P. Chaurand, S. A. Schwartz, and R. M. Caprioli, Curr. Opin. Chem. Biol., 6, 676 (2002).

5) M. Stoeckli, P. Chaurand, D. E. Hallahan, and R. M. Cap- rioli, Nat. Med., 7, 493 (2001).

6) R. M. Caprioli, T. B. Farmer, and J. Gile, Anal. Chem., 69, 4751 (1997).

7) A. C. Crecelius, D. S. Cornett, R. M. Caprioli, B. Williams, B. M. Dawant, and B. Bodenheimer, J. Am. Soc. Mass Spectrom., 16, 1093 (2005).

8) C. Laurent, D. F. Levinson, S. A. Schwartz, P. B. Harrington, S. P. Markey, R. M. Caprioli, and P. Levitt, J. Neurosci. Res., 613 (2005).

9) P. Chaurand, S. A. Schwartz, and R. M. Caprioli, Anal. Chem., 76, 87A (2004).

10) S. A. Schwartz, M. L. Reyzer, and R. M. Caprioli, J. Mass Spectrom., 38, 699 (2003).

11) T. C. Rohner, D. Staab, and M. Stoeckli, Mech. Ageing Dev., 126, 177 (2005).

12) J. Pierson, J. L. Norris, H. R. Aerni, P. Svenningsson, R. M. Caprioli, and P. E. Andren, J. Proteome Res., 3, 289 (2004).

13) S. A. Schwartz, R. J. Weil, M. D. Johnson, S. A. Toms, and R. M. Caprioli, Clin. Cancer Res., 10, 981 (2004).

14) K. Yanagisawa, B. J. Xu, D. P. Carbone, and R. M. Caprioli, Clin. Lung Cancer, 5, 113 (2003).

15) P. Chaurand, S. Fouchecourt, B. B. DaGue, B. J. Xu, M. L. Reyzer, M. C. Orgebin-Crist, and R. M. Caprioli, Proteomics, 3, 2221 (2003).

16) M. Stoeckli, D. Staab, M. Staufenbiel, K. H. Wiederhold, and L. Signor, Anal. Biochem., 311, 33 (2002).

17) N. Masumori, T. Z. Thomas, P. Chaurand, T. Case, M. Paul, S. Kasper, R. M. Caprioli, T. Tsukamoto, S. B. Shappell, and R. J. Matusik, Cancer Res., 61, 2239 (2001).

18) P. Chaurand, B. B. DaGue, R. S. Pearsall, D. W. Threadgill, and R. M. Caprioli, Proteomics, 1, 1320 (2001).

19) M. Rudin, M. Rausch, and M. Stoeckli, Mol. Imaging Biol., 7, 5 (2005).

20) M. Rudin, P. Allegrini, N. Beckmann, H. U. Gremlich, R. Kneuer, D. Laurent, M. Rausch, and M. Stoeckli, Ernst Schering Res. Found. Workshop, 47 (2004).

21) D. Touboul, F. Halgand, A. Brunelle, R. Kersting, E. Tallarek, B. Hagenhoff, and O. Laprevote, Anal. Chem., 76, 1550 (2004).

22) H. Nygren, B. R. Johansson, and P. Malmberg, Microsc. Res. Tech., 65, 282 (2004).

23) P. Sjovall, J. Lausmaa, H. Nygren, L. Carlsson, and P. Malmberg, Anal. Chem., 75, 3429 (2003).

24) I. Ohtsu, T. Nakanisi, M. Furuta, E. Ando, and O. Nishimura, J. Proteome Res., 4, 1391 (2005).

25) T. Nakanishi, I. Ohtsu, M. Furuta, E. Ando, and O. Nishimura, J. Proteome Res., 4, 743 (2005).

26) A. J. Sloane, J. L. Duff, N. L. Wilson, P. S. Gandhi, C. J. Hill, F. G. Hopwood, P. E. Smith, M. L. Thomas, R. A. Cole, N. H. Packer, E. J. Breen, P. W. Cooley, D. B. Wallace, K. L. Williams, and A. A. Gooley, Mol. Cell. Proteomics, 1, 490 (2002).

27) R. L. Martin and F. L. Brancia, Rapid Commun. Mass Spectrom., 17, 1358 (2003).

28) H. Y. Hong, G. S. Yoo, and J. K. Choi, Electrophoresis, 21, 841 (2000).

29) N. Takahashi, A. Roach, D. B. Teplow, S. B. Prusiner, and L. Hood, Cell, 42, 139 (1985).

30) B. Alberts, A. H. Johnson, J. Lewis, M. Raff, K. Roberts, and P. Walter, "MOLECULAR BIOLOGY OF THE CELL," Garland Science, New York (2002), Chap. 2, p. 207.

31) S. Westermann and K. Weber, Nat. Rev. Mol. Cell Biol., 4, 938 (2003).

32) M. Setou, T. Nakagawa, D. H. Seog, and N. Hirokawa, Science, 288, 1796 (2000). 
33) C. Zhao, J. Takita, Y. Tanaka, M. Setou, T. Nakagawa, S. Takeda, H. W. Yang, S. Terada, T. Nakata, Y. Takei, M. Saito, S. Tsuji, Y. Hayashi, and N. Hirokawa, Cell, 105, 587 (2001).

34) T. Nakagawa, M. Setou, D. Seog, K. Ogasawara, N. Dohmae, K. Takio, and N. Hirokawa, Cell, 103, 569 (2000).

35) M. Setou, D. H. Seog, Y. Tanaka, Y. Kanai, Y. Takei, M.
Kawagishi, and N. Hirokawa, Nature, 417, 83 (2002).

36) S. Shimma and M. Setou, Hyoumenkagaku, 27, 79 (2006).

37) K. Ogawa, S. Takeuchi, H. Izumi, T. Harada, Y. Yoshida, and M. Setou, Shimadzu Hyoron, 62, 125 (2006).

Keywords: In situ proteomics, Direct MS/MS, Tissue section, Protein identification, Chemical inkjet technology, Denaturation, Enzymatic digestion 